analysis was used to assess differences in survival (at 30 days, 1 year, and 3 years) by smoking status and gender after adjustment for confounding factors.

Results There were 2726 STEMI patients (26.4\% female) during the study period. Male patients were younger than females $(61.0 \pm 12.1$ vs $65.5 \pm 13.1$ years, $\mathrm{p}=0.02)$. Smoking prevalence was similar in both genders (males $48.3 \%$, females $48.0 \%$ ), but a greater proportion of females had never smoked $(27.8 \%$ vs $21.0 \%, \mathrm{p}=0.01)$. Male current smokers had a significantly worse 3 year mortality than never smokers (HR 1.51, 95\% CI 1.04-2.21, $\mathrm{p}=0.03)$. In female patients smoking status had no significant effect on survival $(\mathrm{p}=0.58)$, which could potentially be due to the modest number $(n=723)$ of female patients in this study. Overall survival was similar in both males and females $(p=0.72)$.

Conclusions There was no evidence of a smoker's paradox in STEMI patients followed up to 3 years in either males or females.

\section{ANGIOGRAPHY AFTER CABG SURGERY; SOLVING THE PROBLEM OF MISSING SURGICAL RECORDS WITH A STERNAL WIRE CODE}

David Wald*, Ben Wald, Alex Shipolini. St Bartholomew's Hospital

10.1136/heartjnl-2017-311726.61
Background The recommendation that surgical records (graft notes) are obtained before coronary angiography in patients with previous coronary artery bypass graft (CABG) surgery is not always followed. Lack of prior information on the number and origin of grafts increases procedure time, complexity and risk to patients. Here, we determine the proportion of angiograms undertaken without graft notes, and propose a code to permanently provide this information.

Methods For patients undergoing angiography at the Barts Heart Centre, London (September 2015-September 2016), records of patients with previous CABG were retrieved. From the procedure reports, we identified patients without graft notes and the indication for the angiogram.

Results Of 6483 patients undergoing angiography, 559 had undergone previous CABG surgery; graft notes were unavailable in $91 / 559$ patients (16\%). In $88 / 91$ patients (97\%), angiography was an unplanned emergency procedure; 84 acute coronary syndromes and 4 out-of-hospital arrests. Three patients had planned angiograms for stable angina. In 20/91 patients additional imaging investigations were required because of uncertainty over whether all grafts had been identified and one patient consequently developed pulmonary oedema due to a large contrast load.

Conclusion About 1 in 6 patients with previous CABG surgery, who require coronary angiography, undergo this procedure without graft notes, almost all in the emergency setting. A clinical code, based on the orientation of the wires used to close the sternum after CABG, could provide a permanent

Sternal wire code for recording number and origin of coronary artery bypass grafts

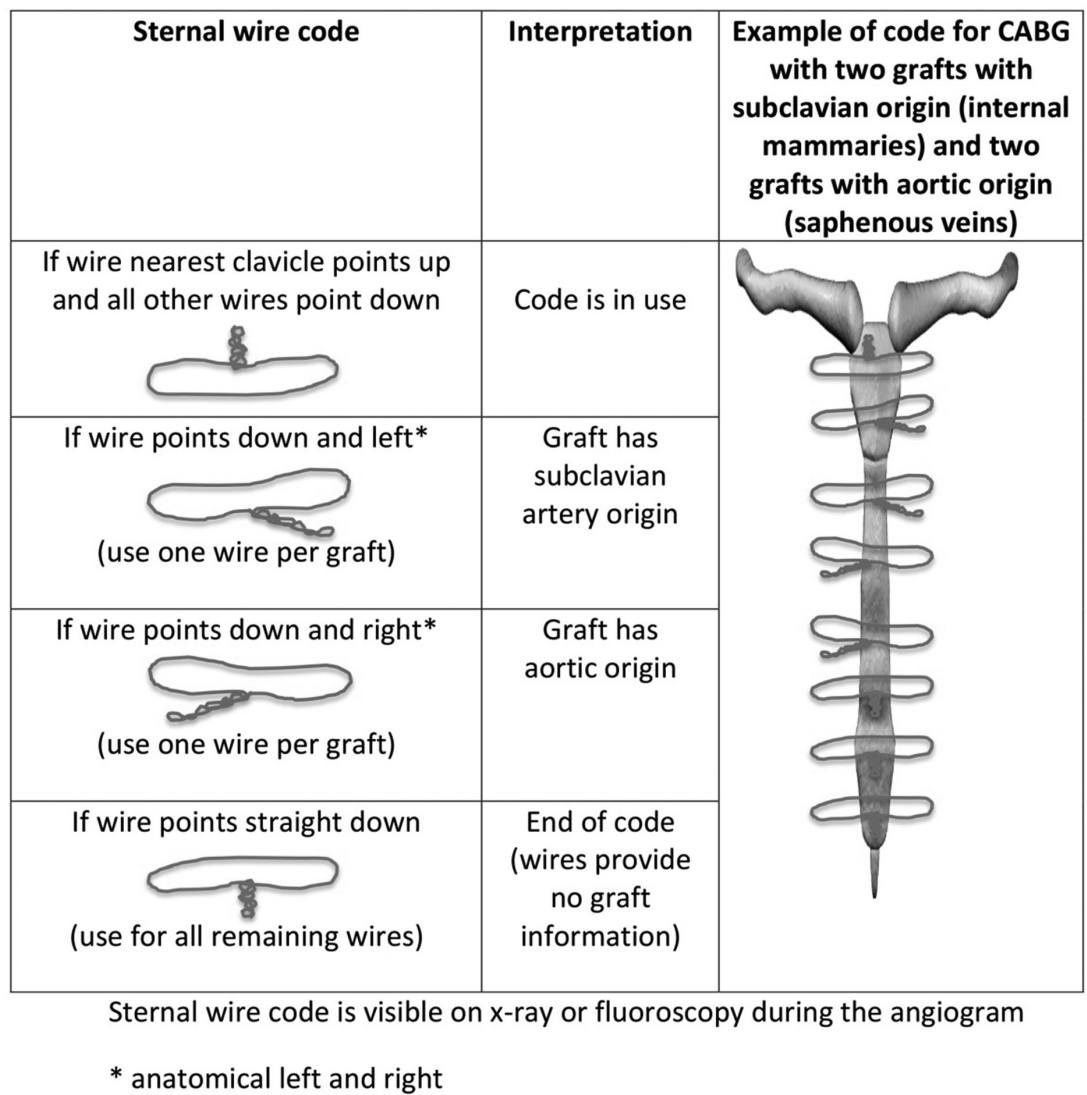


record of the number and origin of the surgical grafts (see Table). We propose that this code, which requires no new technology or technique, be adopted routinely in CABG surgery.

\section{INCREASED RISK OF ACUTE ST-SEGMENT MYOCARDIAL INFARCTION IN FEMALE SMOKERS - A CONTEMPORARY DEMOGRAPHIC STUDY}

\begin{abstract}
${ }^{1}$ James Palmer*, ${ }^{1}$ Amelia Lloyd, ${ }^{1}$ Lloyd Steele, ${ }^{2} J a m e s$ Fotheringham, ${ }^{3}$ Dawn Teare, ${ }^{4}$ Javaid Iqbal, ${ }^{4}$ Ever Grech. 'University of Sheffield Medical School; ${ }^{2}$ University of SHeffield School of Health and Related Research; ${ }^{3}$ University of Sheffield School of Health and Related Research; ${ }^{4}$ South Yorkshire Cardiothoracic Centre
\end{abstract}

\subsection{6/heartjnl-2017-311726.62}

Background Studies have shown that smoking increases the risk of acute ST-segment elevation myocardial infarction (STEMI). However, the impact of gender on this risk is unknown.

Methods This retrospective ecological cohort study examined all patients presenting with acute STEMI undergoing primary percutaneous coronary intervention at the South Yorkshire Cardiothoracic Centre (UK) between 2009-14. Index cases were compared to population data from the UK Office for National Statistics for smoking status, gender and age. Incidence rates of STEMI for current and non-smokers were calculated by gender and their associated 95\% confidence intervals (CI) determined from the Poisson distribution. Agestandardised incidence rate ratios (IRR) comparing STEMI rates between smokers and non-smokers were calculated between genders in three age groups (18-49, 50-64,>65).

Results There were 2,996 STEMI patients. 27.1\% were female, who were significantly older than male patients (mean age: 66.3 vs $60.9, p=0.03$ ). Risk factor prevalence was similar between genders, although hypertension was more common in females $(44.9 \%$ vs $36.7 \%, \mathrm{p}<0.001)$. In current smokers, the peak STEMI rate in females was in the 70-79 age range $(233 / 100,000 \mathrm{pt} / \mathrm{yrs})$ and the 50-59 (458/100,000 pt/yrs) in males. Across all ages, smokers had a significantly higher acute STEMI risk with an IRR of 5.11 (CI: 4.83-5.40). Compared to their non-smoking gender counterparts, females had a significantly higher IRR than males (6.62 (CI: 5.91-7.38) vs 4.46 (CI: 4.18-4.76)), and this difference was significant in all three age groups. The highest IRR was in female smokers in the 18-49 group at 12.67 (CI: 9.69-16.28), vs 8.47 (CI: 7.50-9.53) in males. The most striking difference between genders was in the 50-64 group (IRR 11.03 (CI: 9.33-12.96) vs 4.63 (CI: 4.21-5.08)).

Conclusion Smoking status had a significantly differential effect between genders, with female smokers at increased risk of acute STEMI over male smokers by a factor of 1.48. Young female smokers (18-49) had the highest IRR and were over 12 times more likely to suffer an acute STEMI than their non-smoking peers. The largest IRR gender difference, by a factor of 2.38, was in the 50-64 age group.

\section{OLDER (HIGHER RISK) PATIENTS WITH NON-ST- ELEVATION ACUTE CORONARY SYNDROME (NSTEACS) HAVE THE MOST TO GAIN FROM INVASIVE THERAPY: A POPULATION STUDY IN SOUTH WALES 2004-2014}

${ }^{1}$ Majd Protty*, ${ }^{2}$ Dave Smith, ${ }^{3}$ Arron Lacey, ${ }^{4}$ Phillip Freeman. ${ }^{1}$ Cardiff University; ${ }^{2}$ Morriston Hospital; ${ }^{3}$ Swansea University; ${ }^{4}$ Aalborg University Hospital

\subsection{6/heartjnl-2017-311726.63}

Background and aim Cardiovascular disease is the biggest killer in Wales, with non-ST-elevation acute coronary syndrome (NSTEACS) forming the commonest diagnosis. NSTEACS patients are at risk of death, reinfarction and ischaemia, a risk that increases as cardiovascular risk (GRACE) increases. Standard initial medical therapy followed by the consideration of invasive therapy depending on risk is a proven cost-effective therapy. However, data from national registries suggest that at least 60,000 NSTEACS patients per year do not undergo revascularisation, despite being at high risk. The majority of these patients are elderly and thus high risk (in terms of GRACE score), very few of the trials that shape our guidelines include elderly high risk patients. The aim of our study is to identify factors that influence selection for invasive therapy, and to investigate the consequences on net adverse cardiac outcomes (NACE): adjusted mortality, reinfarction, stroke and significant bleeding.

Methods Using the Secure Anonymized Information Linkage (SAIL) databank, we linked hospital data for all patients admitted to hospitals in South Wales (UK) with a first diagnosis of NSTEACS between 2004-2014. Cohorts of patients undergoing medical versus invasive therapy were identified and validated by a combination of ICD-10 codes, operation codes, and linkage to coronary angiography data. Multivariate analysis was performed to investigate the influence of baseline characteristics on treatment decisions. Propensity matching for a wide variety of baseline characteristics - was carried out with subsequent Cox regression analysis to show the adjusted effects of invasive therapy versus medical therapy only.

Results There were a total of 57,964 NSTEACS patients in the study period. Of these, 20421 received invasive therapy and 37543 received medical therapy only. Medical therapy patients were older (mean age \pm standard deviation 74.9 \pm 13.2 vs $65.0 \pm 11.7$ ) and more likely to have cancer, anaemia, dementia, heart failure, chronic lung, liver and kidney disease $(p<0.05)$. Likelihood of undergoing invasive therapy was significantly reduced in patients over the age of 70 (OR 0.62 in $70-80,0.18$ in $80-90,0.03$ in $90+, \mathrm{p}<0.05)$. After propensity matching between cohorts, net adverse cardiac events (mortality, stroke, reinfarction, significant bleeding) were significantly improved for the invasively managed patients in all age groups. Reduction in NACE were much larger in the elderly high risk groups that received invasive therapy.

Conclusion Our study suggests that older higher risk patients have very low rates of invasive therapy but appear to have more to gain from this approach (than younger, lower risk patients), in terms of mortality and morbidity. In the older higher risk population these results suggest that increased frequency of invasive therapy will lead to reduced short (1 year) and long (5 year) term mortality and net adverse cardiac events (NACE). 